\title{
Acousto-optical multiple interference devices
}

\author{
M. Beck \\ Paul-Drude-Institut für Festkörperelektronik, Hausvogteiplatz, 5-7, 10117 Berlin, Germany \\ M. M. de Lima, Jr. \\ Paul-Drude-Institut für Festkörperelektronik, Hausvogteiplatz, 5-7, 10117 Berlin, Germany \\ and Materials Science Institute, University of Valencia, P.O. Box 22085, E-46071 Valencia, Spain \\ P. V. Santos ${ }^{\text {a) }}$ \\ Paul-Drude-Institut für Festkörperelektronik, Hausvogteiplatz. 5-7, 10117 Berlin, Germany
}

(Received 21 June 2007; accepted 13 October 2007; published online 10 January 2008)

\begin{abstract}
We present a new concept for waveguide acousto-optical devices based on coupled Mach-Zehnder interferometers driven by acoustic waves. These acousto-optical multiple interference devices use the periodic refractive index modulation induced by the acoustic wave to realize functionalities such as ON/OFF switching for an arbitrary time interval, as well as for efficient light modulation at high harmonics of the acoustic frequency and pulse shaping for, e.g., integrated Q-switches. We also discuss application of the concepts to light modulation by very high acoustic frequencies, where the acoustic wavelengths become much shorter than the optical ones. (C) 2008 American Institute of Physics. [DOI: 10.1063/1.2821306]
\end{abstract}

\section{INTRODUCTION}

Control of light beams through acousto-optical interaction normally requires a match in phase between the optical and acoustic waves. In conventional Bragg cells, phase matching is achieved by tuning the angle between the acoustic and optical beams in order to obtain constructive interference of the light reflected at the acoustic wave fronts. In most applications the acoustic wavelengths are much larger than the optical one, so that the phase matching is accomplished under large angles (i.e., close to $90^{\circ}$ ) between the optical and acoustic beams. These large angles become a serious constraint for application of the Bragg cell concepts for acoustooptic-based optical modulation and switching in planar waveguide (WG) structures. Various approaches have been proposed to overcome this limitation based on, e.g., acoustically induced coupling between neighboring $\mathrm{WGs}^{1}$ or the use of photonic structures. ${ }^{2-5}$

In this manuscript, we discuss in detail the alternative concept for WG acousto-optical devices demonstrated by Beck et al., ${ }^{6}$ which is based on a recently realized acoustically driven Mach-Zehnder interferometer (a-MZI). ${ }^{7}$ The use of acoustic waves for the modulation of MZIs was originally proposed by Gorecki et al. ${ }^{8,9}$ The basic idea is illustrated in Fig. 1(a). Here, the strain field of a surface acoustic wave (SAW) modulates the refractive index of the MZI arms, which are oriented perpendicular to the SAW propagation direction. The periodic modulation of the refractive index results in a periodic change of the transmitted light intensity. In the original concept of Ref. 9, only one of the arms is subjected to the acoustic modulation. In order to increase the modulation efficiency, de Lima et al. ${ }^{7}$ proposed the simultaneous modulation of the refractive index of both MZI arms with opposite phase using a single SAW beam. The latter is

${ }^{a)}$ Electronic mail: santos@pdi-berlin.de. implemented by simply choosing the separation between the two MZI arms to be an odd multiple of the half acoustic wavelength $\lambda_{\text {SAW }}$, as illustrated in Fig. 1(a). This procedure effectively doubles the modulation efficiency for a given acoustic strain level. Since the SAW strain field is proportional to the square root of the acoustic power, the required acoustic power is reduced, therefore, by a factor of four. By combining the simultaneous modulation of both arms with narrow and intense acoustic beams generated by focusing interdigitated acoustic transducers (IDTs), ${ }^{7,10}$ compact modulators with a modulated arm length of only $\approx 15 \mu \mathrm{m}$ have been demonstrated on the (Al,Ga)As material system. This acousto-optical modulation approach can be easily transferred to other material systems, such as silicon-on-insulator (SOI) and InP-based WGs. Also, although the discussion will be based on acousto-optical modulation, the concepts can also be extended to light control using other wavelike excitations such as magnetostatic ${ }^{11}$ and plasmons.

The a-MZI in Fig. 1(a) operates as an efficient light modulator when the static optical phase difference $\left(\phi_{s}^{\mathrm{opt}}\right)$ between the arms, which is controlled by their optical lengths, equals $\pi / 2$. In this case, the amplitude modulation of the
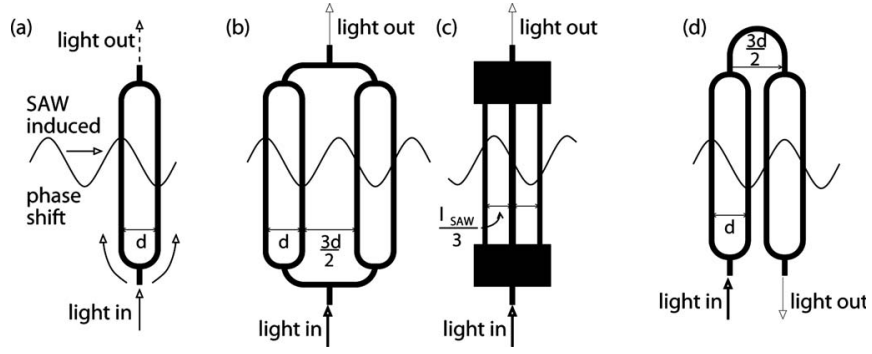

FIG. 1. Illustrations of the (a) SAW-driven Mach-Zehnder interferometer with separation $d=\lambda_{\mathrm{SAW}} / 2$ between the arms (as described in Ref. 7). Proposed devices in the parallel (P) configuration with (b) $N_{P}=4$ and (c) $N_{P}$ $=3$ arms connected in parallel. (d) Device in the serial (S) configuration consisting of $N_{S}=2$ Mach-Zehnder interferometers connected in series. 

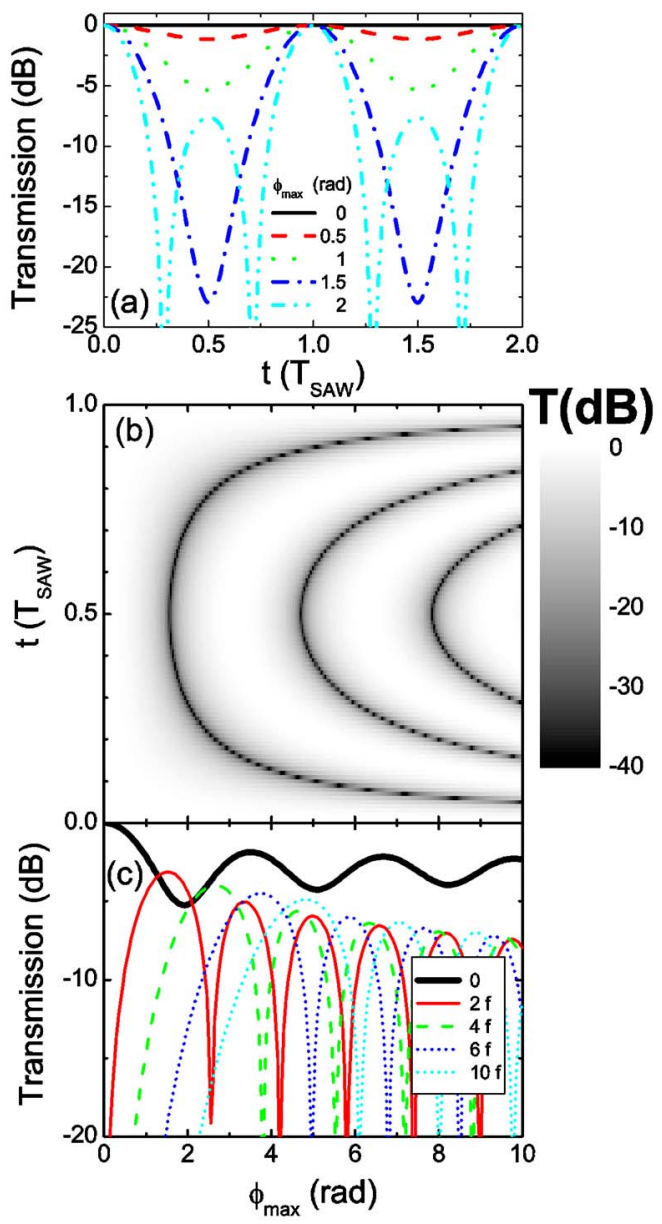

FIG. 2. (Color online) (a) Dependence of the optical transmission through an ideal a-MZI as a function of the phase modulation amplitude $\phi_{\max }$. The time scale is in units of the SAW period $T_{\mathrm{SAW}}$. (b) Gray-scale representation of the transmission as a function of $\phi_{\max }$ (horizontal axis) and of time (vertical axis). (c) Fourier decomposition of the transmission into its Fourier components. The dark solid line (denoted as 0 ) corresponds to the timeaveraged transmission. The numbers denote the order of the harmonic of the SAW frequency.

SAW driving signal will be transferred to the MZI light transmission intensity in the form of a periodic modulation at the SAW frequency $f_{\mathrm{SAW}}$, with side-bands corresponding to the actual modulation signal. A second functionality offered by the acoustic MZI is the efficient generation of light beams modulated at harmonics of the SAW frequency $\left(f_{\mathrm{SAW}}\right)$. The best performance for transmission modulation at twice the acoustic frequency is achieved by using a perfectly symmetric device (i.e., for a static optical phase shift $\phi_{\mathrm{opt}}=0$ ). The light transmission $T_{\text {MZI }}\left(\phi_{\max }\right)$ for such a device is given by

$$
T_{\mathrm{MZI}}\left(\phi_{\max }\right)=\frac{1}{2}\left\{1+\cos \left[2 \phi_{\max }\left(\sin \omega_{\mathrm{SAW}} t\right)\right]\right\},
$$

where $\omega_{\mathrm{SAW}}=2 \pi f_{\mathrm{SAW}}$ is the SAW angular frequency and $\phi_{\max }=2 \pi \Delta n \ell / \lambda$ denotes the amplitude of the light phase change in each one of the arms. $\phi_{\max }$ is stated in terms of the arm length $\ell$ and the amplitude $\Delta n$ of the refractive index modulation induced by the SAW for a light beam with wavelength $\lambda$. The modulation of the transmission at $2 f_{\mathrm{SAW}}$ becomes evident in the time dependence of the transmission calculated from Eq. (1) for different phase shift amplitudes $\phi_{\max }$ as illustrated in Fig. 2(a). The gray-scale plot of Fig. 2(b) shows an alternative representation of the transmission over a wide range of phase amplitudes $\phi_{\max }$, where the vertical and horizontal scales correspond, respectively, to time and $\phi_{\max }$. The dark areas represent regions of reduced transmission. For operation under a fixed phase $\phi_{\max }$ (corresponding to the time dependence along a vertical line of the plot), the transmission is modulated at even multiples of $f_{\text {SAW }}$, with minima corresponding to the dark regions. Although its average value reduces under acoustic excitation, the transmission will always contain many even harmonics of the fundamental modulation frequency $2 f_{\text {SAW }}$, as shown in the harmonic decomposition in Fig. 2(c). A minimum average transmission $T \approx-5.25 \mathrm{~dB}$ is obtained for $\phi_{\max } \approx 1.9 \mathrm{rad}$ [cf. thick line in Fig. 2(c), which yields the average transmission through the device]. Note, however, that the transmission always reaches unity twice during each SAW cycle, independent of the modulation phase $\phi_{\max }$.

In contrast to modulation and harmonic generation, switching applications demand the suppression of the transmitted (or reflected) light intensity for an arbitrary time interval. Due to the periodic nature of the acoustic modulation, this functionality cannot be realized using the simple structure of Fig. 1(a). The same applies to signal processing applications where one wants to control the intensity of the transmitted light. These restrictions can be easily overcome if one operates with light pulses much shorter than the SAW period. In this case, efficient switching and pulse intensity control can be realized using the simple a-MZI of Fig. 1 by synchronizing the light pulses with the SAW phase.

In this paper, we describe a new class of WG acoustooptical devices (denoted acousto-optical multiple interference devices, AOMID) based on the modulation of MZI interferometers with multiple arms by an acoustic wave. These devices use the periodic modulation of WG structures by acoustic waves to realize (a) ON/OFF switches for continuous wave (cw) light beams with arbitrary "ON" and "OFF" times [as opposed to the modulation at the acoustic frequency demonstrated for the acoustic MZI of Fig. 1(a)]; (b) harmonic generators yielding light beams modulated at a multiple of the acoustic frequency; and (c) pulse shapers for the generation of short light pulses. Due to the strength of the acousto-optic interaction, the interaction length between the optical and acoustic waves in AOMIDs is expected to be much shorter than for electro-optical devices. In addition, AOMIDs are compatible with integrated optics components and can be fabricated using conventional planar technology. Finally, since the operation principle relies on the elastooptical effect, the concept applies to a wide range of material systems including, for instance, SOI and WGs based on amorphous materials, where linear electro-optical effects are forbidden by symmetry.

In the following section (Sec. II), we discuss the design of AOMIDs as well as the framework for the calculation of the optical transmission through the proposed WG structures. Section III A analyzes the dependence of the calculated optical transmission on the optical phase shift induced by the acoustic wave for different device configurations. The performance of the proposed devices for ON/OFF switching and for high harmonic generation are discussed in Secs. III B and 
III C, respectively. A further approach to modulate light with very high acoustic frequencies will be presented in Sec. IV. The main conclusions of the manuscript are summarized in Sec. V.

\section{DEVICE CONCEPTS}

The functionalities of the AOMIDs rely on the control of the transmitted intensity of a continuous wave $(\mathrm{cw})$ light beam by the SAW intensity. Although the acoustic wave reduces the average transmission through the simple a-MZI of Fig. 2, the transmitted light still reaches unity twice within each SAW cycle. The simultaneous reduction of the average value as well as of the amplitude of the transmission oscillations can be achieved by increasing the number of arms $(N)$ in the interferometer. As will be discussed in detail below, the arms can be connected in parallel [configuration "P," cf. Figs. 1(b) and 1(c)] or in series [configuration "S," cf. Fig. 1(d)]. In order to analyze the transmission properties of these devices, we will make the following simplifying assumptions:

1. All interferometer arms have the same optical path length in the absence of acoustic excitation, i.e., all optical phase differences vanish for all paths between input and output (or are multiples of $2 \pi$ ). Hence, all optical beams interfere constructively and the devices have unitary transmission when the SAW is turned off. ${ }^{12}$

2. In each arm, the light experiences a SAW-induced sinusoidal phase modulation with the same amplitude $\phi_{\max }$.

3 . The arm widths are much smaller than the SAW wavelength $\lambda_{\mathrm{SAW}}$, so that the SAW-induced refractive index modulation $\Delta n$ can be considered constant across the arm cross section. Concepts to overcome this constraint will be discussed in Sec. IV.

4. The light propagation time through each arm (given by $d /\left(n_{\text {eff }} c_{0}\right)$, where $c_{0}$ is the light speed in vacuum, $d$ the arm length, and $n_{\text {eff }}$ the effective refractive index of the WG) is much shorter than $T_{\mathrm{SAW}} / N$.

In the $\mathrm{P}$ devices, $N_{P} \geq 2$ interferometer arms are connected in parallel, as illustrated for $N_{P}=4$ and $N_{P}=3$ in Figs. 1(b) and 1(c), respectively. The arms are spaced in such a way that they experience SAW phases differing by $2 \pi m / N_{P}$ $\left(m=1, \ldots, N_{P-1}\right)$, i.e., the SAW phases of the $N_{P}$ arms are equally spaced within $2 \pi$. This SAW phase distribution is essential for the operation of the devices. The most obvious way to arrange the WGs is by choosing a separation of $\lambda_{\mathrm{SAW}} / N_{P}$ between the center positions of adjacent arms. Note, however, that the phase relationship remains unchanged if the distance between the arms is changed by a multiple of $\lambda_{\text {SAW }}$. The device in Fig. 1(b) has $N_{P}=4$ and consists of two a-MZIs connected in parallel and separated by $5 \lambda_{\mathrm{SAW}} / 4$. This design uses symmetric Y-shaped (or T-shaped) WG splitters to ensure equal light phase shifts through each of the arms, an approach that can be extended to devices with $N_{P}=2^{m}$ arms ( $m$ is an integer). Devices with an arbitrary number of arms can be realized, e.g., using multimode interference (MMI) couplers, ${ }^{13}$ as illustrated in Fig. 1(c) for $N_{P}=3$. The requirement of equal light phase shifts in the absence of a SAW can be satisfied by using WGs of different widths (as indicated in the figure, where the center arm is thicker than the outer ones), leading to different effective refractive indices for the optical modes.

It is easy to see from Fig. 1(b) that the total transmission becomes invariant with respect to shifts of the SAW phase by $2 \pi / N_{P}$. As a result, when the refractive index of the arms is modulated by a SAW with frequency $f_{\mathrm{SAW}}$, the transmitted intensity will contain only harmonics of the fundamental frequency $N_{P} f_{\text {SAW }} \cdot{ }^{14}$ With the above-mentioned assumptions, the optical transmission coefficient is obtained by adding the transmitted field through the arms according to

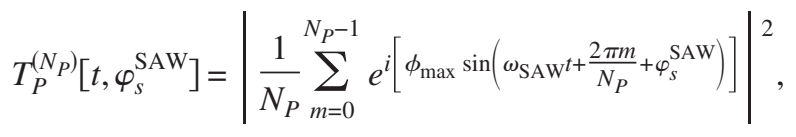

where $\varphi_{s}^{\mathrm{SAW}}$ is the phase of the SAW at the first interferometer $\operatorname{arm}\left(\varphi_{s}^{\mathrm{SAW}}\right.$ depends on the distance between the transducer and the interferometer arms). This factor does not affect the transmission amplitude in the $\mathrm{P}$ configuration but will be important for $\mathrm{S}$ devices, which are discussed in the following.

In the $\mathrm{S}$ configuration, $\mathrm{P}$ devices are connected in series and arranged to be modulated at different phases of the acoustic wave. The corresponding design for $N_{S}=2$ interferometers connected in series, each with $N_{P}=2$ arms [i.e., corresponding to the a-MZIs in Fig. 1(a)], is illustrated in Fig. 1(d). As in the parallel configuration, the lateral separations between the a-MZIs is chosen such that the SAW-induced phase shifts in the individual arms are evenly distributed within the $2 \pi$ SAW phase range [e.g., by an equidistant phase spacing equal to $2 \pi /\left(N_{S} N_{P}\right)$ ]. The transmission intensity is given by

$$
T_{S}^{\left(N_{P}, N_{S}\right)}[t]=\prod_{m=0}^{N_{S^{-1}}} T_{P}^{\left(N_{P}\right)}\left[t, \frac{2 \pi m}{N_{S} N_{P}}\right] .
$$

Arbitrary combinations of $N_{S}$ and $N_{P}$ are possible, and also devices with unequal numbers of parallel arms may be connected in series. For simplicity, in the following we will restrict the discussion to (i) $\mathrm{P}$ devices consisting of $N_{P}$ arms (i.e., $N_{S}=1$ ) and (ii) $\mathrm{S}$ devices consisting of $N_{S}$ a-MZIs connected in series (i.e., $N_{P}=2$ ).

\section{RESULTS}

\section{A. Optical response}

The gray scale plots of Figs. 3(a) and 3(b) compare the transmission (in decibels) of $\mathrm{P}$ and $\mathrm{S}$ devices with a total number of four arms \{corresponding to $N=N_{P} \times N_{S}=4 \times 1$ [Fig. 3(a)] and $N=N_{P} \times N_{S}=2 \times 2$ [Fig. 3(b)]\}, respectively. As in Fig. 2(b), dark areas indicate regions of reduced transmission and the horizontal and vertical scales represent the modulation amplitude $\phi_{\max }$ (horizontal scale) and time (in units of the SAW period $T_{\mathrm{SAW}}$ ), respectively. The corresponding plots for devices with a total of eight arms (i.e., with $N=N_{P} \times N_{S}=8 \times 1$ and $\left.N=N_{P} \times N_{S}=4 \times 2\right)$ are shown in Figs. 3(c) and 3(d), respectively. ${ }^{15}$ 


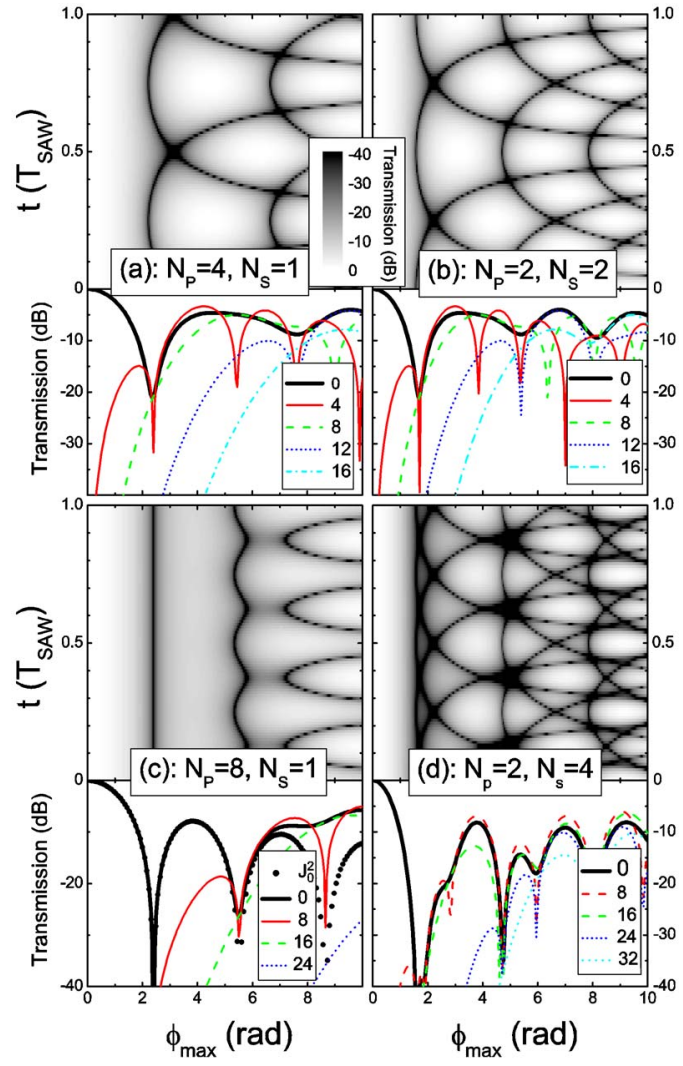

FIG. 3. (Color online) Transmission of devices with a total number of four [(a) and (b), top panels] and eight [(c) and (d), bottom panels] interferometer arms, respectively. The left panels show results for the $\mathrm{P}$ configuration with (a) $N_{P}=4$ and (c) $N_{P}=8$ arms. The right panels $\mathrm{S}$ display the transmission for $\mathrm{S}$ devices with (b) $N_{S}=2$ and (d) $N_{S}=4$. In each panel, the upper plot shows a gray-scale representation of the transmission as a function of the modulation amplitude $\phi_{\text {SAW }}$ (horizontal scale) and time (vertical scale). The lower plot in each panel displays the Fourier components of the transmitted intensity for various multiples of the SAW frequency (indicated in the legend), with the thick solid lines denoted as " 0 " corresponding to the average transmission. The dots in the lower plot of (c) show the Bessel function $J_{0}^{2}\left(\phi_{\mathrm{SAW}}\right)$, which essentially coincides with the average transmission for $\phi_{\max }<6 \mathrm{rad}$.

The number of low-transmission regions increases with the number of arms and with $\phi_{\max }$, thus leading to a higher harmonic content. The latter is shown in the harmonic decomposition displayed in the diagrams below each gray-scale plot. Here, the zero frequency component (indicated by the thick solid line) corresponds to the average (cw) transmission through the device. Note that the fundamental modulation frequency is equal to the SAW frequency multiplied by the total number of arms. In each case, the fundamental modulation component with frequency $N f_{\mathrm{SAW}}$ and up to three higher harmonics are shown by the thin lines.

The distribution of harmonic components is qualitatively different for the $\mathrm{P}$ and $\mathrm{S}$ configuration. While the transmission of the $\mathrm{P}$ configuration for a given modulation phase $\phi_{\max }$ is normally dominated by a single harmonic component, the response of the $\mathrm{S}$ configuration contains several harmonics of comparable amplitude [see lower plots in each of the panels in Fig. 3(c)]. Note, for instance, that the amplitudes of the 8th and 16th harmonics for $\phi_{\mathrm{SAW}} \approx 4 \mathrm{rad}$ are comparable to the average transmission (zeroth order) for the device with $N=8$ in the $\mathrm{S}$ configuration [Fig. 3(d)]. For the $\mathrm{P}$

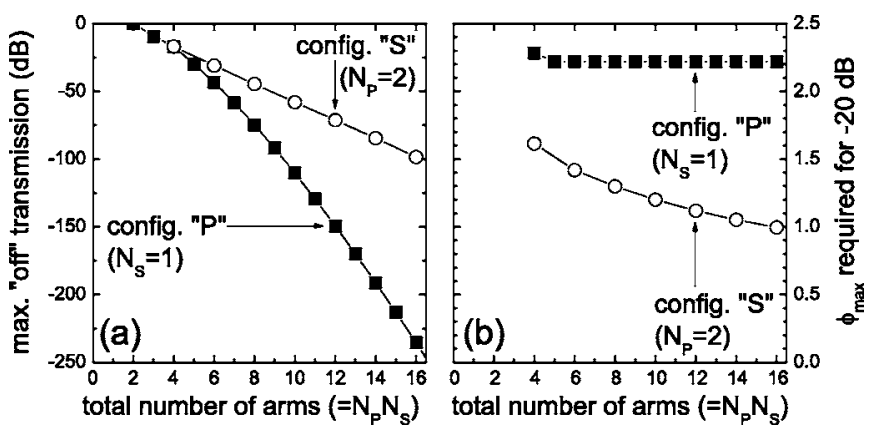

FIG. 4. (a) Maximum residual transmission in the OFF state and (b) phase shift amplitude $\phi_{\max }$ per interferometer arm required to achieve a $20 \mathrm{~dB}$ suppression of the averaged transmission intensity calculated for $\mathrm{P}$ and $\mathrm{S}$ devices with the same total number of arms $N=N_{S} \times N_{P}$. For $N<4,-20 \mathrm{~dB}$ transmission cannot be achieved.

configuration, in contrast, the corresponding amplitudes of the 16th harmonic for the same phase is much smaller. This behavior is also reflected in the larger number of dark regions for a particular phase $\phi_{\max }$ in the gray scale plots for $\mathrm{S}$ devices in Figs. 3(c) and 3(d).

\section{B. Acoustic switches}

Operation as an ideal ON/OFF switch requires that the total transmission drops to a small value for a particular SAW amplitude. Ideally, this condition corresponds to a vertical line of vanishing transmission in the gray-scale diagrams of Fig. 3. For the P configuration (left panels in Fig. 3 ), this condition is best satisfied near $\phi_{\max }=2.4 \mathrm{rad}$ [cf. Fig. 3 (c)], where the transmission reduces for all harmonic components. In fact, it can be shown that in the limiting case of $N_{P} \rightarrow \infty$, the average transmission of devices becomes equal to $J_{0}^{2}\left(\phi_{\max }\right)$, where $J_{0}$ is the zeroth-order Bessel function of the first kind. $J_{0}^{2}$ is displayed by the dots in the lower graph of Fig. 3(c). This function has its first zero at $\phi_{\max }$ $=2.40483 \mathrm{rad}$, and reproduces very well the average $(\mathrm{cw})$ transmission of parallel AOMIDs with a large number of arms $\left(N_{P} \geq 8\right)$ in the phase range $\phi_{\max } \leq 6 \mathrm{rad}$. For smaller $N_{P}$, the average transmission also reduces for $\phi_{\max }$ $\approx 2.40 \mathrm{rad}$, but the transmission signal contains harmonic components with significant amplitudes [cf. Fig. 3(a)].

The $\mathrm{S}$ configuration can also be used for ON/OFF switches. As in the P devices, the high-transmission ON state is obtained by switching off the SAW. A low-transmission (OFF) state is achieved for a SAW-induced phase equal to an odd multiple of $\phi_{\mathrm{SAW}}=\pi / 2 \mathrm{rad}$. As can be easily seen in Fig. 1(d), these phase values block the transmission of one of the serial sections, leading to an overall transmission reduction [cf. Fig. 1(d)]. The phase dependence of the transmission for devices with four and eight serial sections is displayed in Figs. 3(b) and 3(d), respectively.

In order to compare the two configurations, Fig. 4(a) displays the residual transmission in the OFF state for $\mathrm{P}$ and $\mathrm{S}$ devices as a function of the total number of arms $N_{S}$ $\times N_{P}$. The residual transmission is defined as the maximum instantaneous transmission during a SAW cycle, which was found to be typically approx. $3 \mathrm{~dB}$ above the average (or $\mathrm{cw}$ ) transmission. The residual transmission is much lower for 
the $\mathrm{P}$ than for the $\mathrm{S}$ configuration for devices with more than three arms. In addition, in the $\mathrm{P}$ configuration the residual transmission reduces much faster with the number of arms, thus making it possible to achieve very high ON/OFF contrast ratios. One disadvantage of the $\mathrm{P}$ configuration, however, is the higher phase modulation amplitude $\left(\phi_{\max }\right.$ $\approx 2.4 \mathrm{rad}$ ) required to reach a given level of transmission suppression as compared to $\mathrm{S}$ devices $\left(\phi_{\max } \approx 1.6 \mathrm{rad}\right)$, which translates into lower acoustic switching powers. The modulation phase for the $\mathrm{S}$ configuration can be further reduced by increasing the number of WGs. This behavior is illustrated in Fig. 4(b), which compares for the two configurations the required number of arms to achieve an average transmission of $1 \%$ in the OFF state (corresponding to a 20 $\mathrm{dB}$ reduction relative to the $\mathrm{ON}$ state). In contrast, for $\mathrm{P}$ devices the required $\phi_{\max }$ values for the OFF state are essentially independent of the number of arms.

For realistic devices, the advantages associated with the higher contrast of the $\mathrm{P}$ configuration as compared to $\mathrm{S}$ may be partially offset by the narrower width of the transmission minima as a function of $\phi_{\max }$ (cf. Fig. 3), which makes the residual transmission of $\mathrm{P}$ devices more sensitive to fabrication errors. The $\mathrm{P}$ configuration, however, is easier to implement using the narrow focused acoustic beams ${ }^{7,10}$ required for compact devices, since a single IDT can drive several arms. A single transducer can also be used for the $\mathrm{S}$ configuration if the serial sections can be connected by U-shaped WGs, as illustrated in Fig. 1(a). This design, however, requires WGs with small curvature radii (on the order of a few acoustic wavelengths), which are more easily implemented in material systems with high refractive index contrast [e.g., SOI and membrane WGs].

\section{Generation of high harmonics and pulses}

So far, we have concentrated on ON/OFF switching applications, where one operates the AOMIDs at a modulation phase yielding minimum transmission. AOMIDs can also deliver other functionalities, such as light modulation at harmonics of the SAW frequency as well as the generation of short light pulses. For these applications, one takes advantage of the fact that AOMIDs generate several harmonics of the fundamental SAW frequency, in particular, for highphase-modulation amplitudes $\phi_{\max }$ [cf. Figs. 1(b) and 1(d)]. The modulation of a $\mathrm{cw}$ input light beam at a particular harmonic of the SAW frequency is best realized in the $\mathrm{P}$ configuration, where the number of high-frequency components contributing to the total modulation is smaller than for the $\mathrm{S}$ configuration. This property can be used for the selective modulation of the transmitted light beam at a particular harmonic of the SAW frequency by an appropriate choice of the number of arms and modulation amplitude. As an example, Fig. 5(a) shows the efficient generation of the 6th harmonic of the SAW frequency using a $\mathrm{P}$ device consisting of three arms modulated with a phase amplitude $\phi_{\max }$ $=4 \pi / 3 \mathrm{rad}$.

Short light pulses can be realized by exploring the high harmonics content of the $\mathrm{S}$ configuration. Figure 5(b) shows the transmission of three a-MZI connected in series and sub-

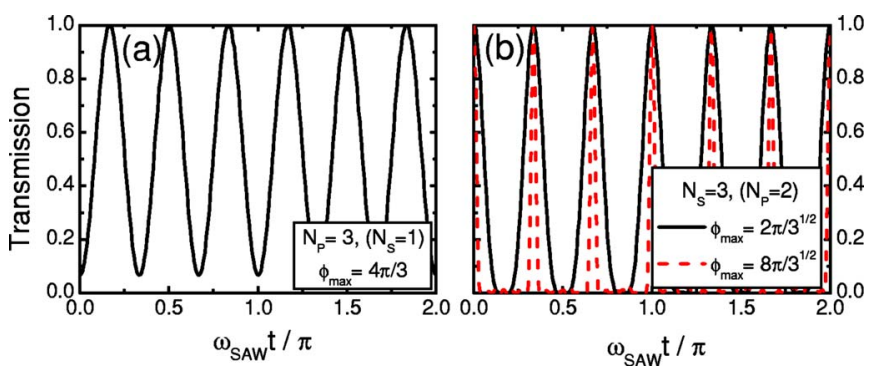

FIG. 5. (Color online) (a) Transmission of a device consisting of three arms connected in parallel, showing the strong modulation at the 6th harmonic of the SAW frequency. (b) Transmission of a device consisting of three a-MZIs connected in series, showing the generation of six short light pulses per SAW period.

jected to modulation amplitudes $\phi_{\max }=2 \pi / \sqrt{3} \mathrm{rad}$ (solid line) and $8 \pi / \sqrt{3} \mathrm{rad}$ (dashed line), respectively. These devices reflect the incoming $\mathrm{cw}$ light beam, except for short periods of time when the transmission approaches unity. Possible applications include output mirrors for integrated lasers capable of generating short light pulses without modelocking (integrated Q-switches). In the illustrated example $\left(N_{S}=3\right.$ and $\left.\phi_{\max }=8 \pi / \sqrt{3} \mathrm{rad}\right)$, repetition rates of $10 \mathrm{GHz}$ and $40 \mathrm{GHz}$ can be realized using SAW frequencies of 1.67 $\mathrm{GHz}$ and $6.67 \mathrm{GHz}$, respectively.

The operation of the AOMIDs requires that the WGs are excited by a traveling SAW beam in order to produce the correct phase modulation difference between the arms. The interdigitated transducers employed for the generation of these beams are, in general, bidirectional devices, which emit SAWs in both directions along their axes. The overall modulation efficiency of the devices can then be increased by using unidirectional transducers, which emit preferentially along one direction.

\section{HIGH ACOUSTIC FREQUENCIES}

In the previous sections, we have implicitly assumed that the width of the WG arms forming the AOMIDs, which is dictated by the optical wavelength and the refractive indices of the materials, is much smaller than the acoustic wavelength $\lambda_{\text {SAW }}$. This condition is normally satisfied for modulators operating in the near-infrared and visible light range using acoustic waves with frequencies up to a few gigahertz. During the last two decades, different approaches have been proposed for the generation of acoustic waves with frequencies up to the terahertz range, where the acoustic wavelengths become much shorter than the optical ones. Examples are the piezoelectric generation of bulk acoustic waves, where frequencies up to $96 \mathrm{GHz}$ have been reached, ${ }^{16}$ as well as the generation of acoustic waves using short laser pulses. In the last case, the generation of acoustic pulses with frequencies in the terahertz range has been demonstrated, as well as their application for optical control in the picosecond time scale. ${ }^{17,18}$

While short acoustic wavelengths are expected to increase the frequency response of acousto-optical devices, novel designs are required for optical control using either the simple a-MZI or the coupled interferometer structures. Figure 6 illustrates one approach compatible with very short 


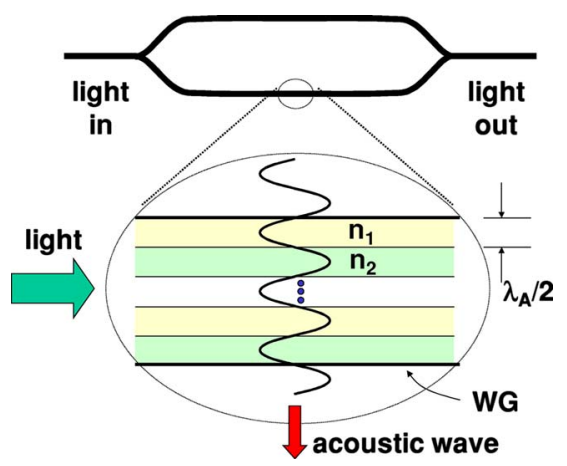

FIG. 6. (Color online) Acousto-optical modulation using structured WGs consisting of layers of materials 1 and 2 with thicknesses corresponding to half an acoustic wavelength $\left(\lambda_{A}\right)$. The modulation amplitude $\delta n_{i}(i=1,2)$ of refractive indices of the two materials induced by the acoustic field are assumed to have different amplitudes (i.e., $\delta n_{1} \gg \delta n_{2}$ ).

acoustic wavelengths based on structured WGs. Here, the core of the optical WGs forming the MZI arms is replaced by a periodic layer stack (a superlattice) with a repetition period much smaller than the light wavelength. We will consider in the following the simplest configuration, where the superlattice consists of two materials (1 and 2 ) with refractive indices $n_{1}$ and $n_{2}$, respectively. For the optical wave, the superlattice is equivalent to a homogeneous effective medium with an effective refractive index $n_{\text {eff }}$ that depends on the light wavelength, polarization, as well as on the optical properties and thicknesses of the constituent layers. For equal layer thicknesses and light polarization in the plane of the layers, the effective refractive index is given by $n_{\mathrm{eff}} \approx \sqrt{\left(n_{1}^{2}+n_{2}^{2}\right) / 2}$. We will further assume that the acoustically induced change in the refractive index of material $1\left(\delta n_{1}\right)$ is much larger than for layer 2 (i.e., $\delta n_{1} \gg \delta n_{2}$ ). This requirement can be fulfilled, for instance, by operating at a photon energy close to an electronic transition of material 1. Under these conditions, the modulation amplitude $\left(\delta n_{\mathrm{eff}}\right)$ of the effective refractive index induced by the acoustic field becomes $\delta n_{\text {eff }}=\frac{1}{2} \frac{n_{1}}{n_{\text {eff }}} \delta n_{1}$. For application, the reduced acoustically induced phase modulation as compared to a simple WG of material 1 $\left(=\delta n_{1}\right)$ can be compensated by using longer WGs.

The superlattice approach becomes especially appealing for acousto-optical modulation by bulk (instead of surface) acoustic waves, if the WGs can be fabricated by growing layers with different dielectric materials. An example of a serial switch using bulk acoustic transducers is illustrated in Fig. 7. Here, each horizontal WG section consists of a layer stack with high effective refractive index separated by clad-

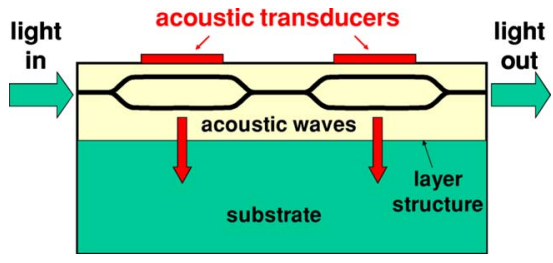

FIG. 7. (Color online) Optical switch consisting of two acoustic MachZehnder modulators in series and modulated by bulk acoustic waves. The superlattice WGs (cf. Fig. 6) in this case were fabricated by growing dielectric layers of different refractive indices. The acoustic waves were generated by transducers deposited on the top of the sample. ding layers with lower index. The Y-splitters are produced by optically interconnecting the two layers. The WGs are modulated by two transducers deposited on the surface of the sample. Configurations using a single transducer are also viable if the depth of the two serial sections can be controlled, thus leading to different SAW phases in the two sections. Alternatively, high-frequency bulk waves can be produced using short laser pulses. ${ }^{17,18}$ We note that Gottlieb and Brandt ${ }^{19}$ have proposed a similar concept for acousto-optical phase shift arrays using bulk acoustic waves to drive a single, unstructured WG: the novelty of the scheme in Fig. 7 results from the combination of superlattice WGs with the multiple interference approach, which allows for efficient ON/OFF switching using very high acoustic frequencies. Finally, since superlattices with well-defined layer thicknesses down to the nanometer range can be produced by wellcontrolled growth techniques, this approach can be applied for the realization of acousto-optic switches and modulators with operation frequencies up to the terahertz range.

\section{CONCLUSIONS}

In conclusion, we have presented a concept for acoustooptical light control in WG structures based on the modulation of the refractive index of MZI interferometers with multiple arms by an acoustic wave. We have discussed the use of these AOMIDs for the realization of optical switches, frequency converters, and pulse shapers and presented a framework for the calculation of their performance. Since the acousto-optical effect is normally stronger than the optical nonlinear and the electro-optical interactions, the devices are expected to be very compact, as recently demonstrated for acoustically driven MZIs. ${ }^{7}$ In order to take advantage of the faster switching times expected from high-frequency acoustic waves, we have also introduced a design for AOMIDs using SAW wavelengths much shorter than the optical ones. Finally, the AOMID concept is compatible with integrated optics based on planar technology and can be applied for different material systems.

\section{ACKNOWLEDGMENTS}

We thank K.-J. Friedland for comments and for a critical reading of the manuscript, as well as $\mathrm{M}$. van der Poel and M. B. Dühring for helpful discussions. Support from the EU Network of Excellence ePIXnet is gratefully acknowledged.

\footnotetext{
${ }^{1}$ A. Korpel, Acousto-Optics (Marcel Dekker, Inc., New York, 1997).

${ }^{2}$ W. L. Liu, P. S. J. Russell, and L. Dong, Opt. Lett. 22, 1515 (1997).

${ }^{3}$ P. S. J. Russell, T. A. Birks, and C. N. Pannell, U.S. Patent No. 5,915,050 (1999).

${ }^{4}$ P. V. Santos, J. Appl. Phys. 89, 5060 (2001).

${ }^{5}$ M. M. de Lima, Jr. and P. V. Santos, Rep. Prog. Phys. 68, 1639 (2005).

${ }^{6}$ M. Beck, M. M. de Lima, Jr., E. Wiebicke, W. Siedel, R. Hey, and P. V. Santos, Appl. Phys. Lett. 91, 061118 (2007).

${ }^{7}$ M. M. de Lima, Jr., M. Beck, R. Hey, and P. V. Santos, Appl. Phys. Lett. 89, 121104 (2006)

${ }^{8}$ C. Gorecki, E. Bonnotte, H. Toshioshi, F. Benoit, H. Kawakatsu, and H. Fujita, Proc. SPIE 3098, 392 (1997).

${ }^{9}$ C. Gorecki, F. Chollet, E. Bonnotte, and H. Kawakatsu, Opt. Lett. 22, 1784 (1997).

${ }^{10}$ M. M. de Lima, Jr., F. Alsina, W. Seidel, and P. V. Santos, J. Appl. Phys. 94, 7848 (2003).
} 
${ }^{11}$ A. D. Fisher, J. N. Lee, E. S. Gaynor, and A. B. Tveten, Appl. Phys. Lett. 41, 779 (1982).

${ }^{12}$ One can also envisage devices where the transmission vanishes in the absence of a SAW.

${ }^{13}$ L. B. Soldano, F. B. Veerman, M. K. Smit, B. H. Verbeek, A. H. Dubost, and E. C. M. Pennings, J. Lightwave Technol. 10, 1843 (1992).

${ }^{14}$ For odd $N_{P}$, the lowest harmonic in the intensity modulation is given by $2 N_{P} f_{\text {SAW }}$, while only phase modulation will occur at odd multiples of $N_{P} f_{\text {SAW }}$.
${ }^{15}$ It can be easily seen by comparing the respective gray scale plots in Figs. 2(b), 3(b), and 3(d) that the transmission for the S devices is the product of the transmission of several a-MZIs modulated at different SAW phases.

${ }^{16}$ B. Hadimioglu, L. J. La Comb, Jr., D. R. Wright, B. T. Khuri-Yakub, and C. F. Quate, Appl. Phys. Lett. 50, 1642 (1987).

${ }^{17}$ C.-K. Sun, J.-C. Liang, and X.-Y. Yu, Phys. Rev. Lett. 84, 179 (2000).

${ }^{18}$ A. Akimov, A. Scherbakov, D. R. Yakovlev, C. T. Foxon, and M. Bayer, Phys. Rev. Lett. 97, 037401 (2006).

${ }^{19}$ M. Gottlieb and G. B. Brandt, U.S. Patent No. 4,395,702 (1983). 